\title{
LINGUAGGIO MENTALE E ATTI DI PENSIERO IN GUGLIELMO DI OCKHAM
}

Francesco Bottin*

SÍNTESE - Guilherme de Ockham desenvolveu temas de gnosiologia que o colocam como termo relativamente fácil de comparação com pensadores modernos. É o caso de seu estudo sobre a linguagem mental, que em muito $o$ aproxima, por exemplo, de certas teorias de Putnam, a respeito da teoria da representação.

PALAVRAS-CHAVE - Filosofia medieval. Ockham. Putnam. Linguagem mental. Verbum mentis. Atos de pensamento. Teoria da representação. Conhecimento direto.
ABSTRACT - William Ockham developed themes of epistemology which place him in position which can easily be compared to that of modern thinkers. Such is notably the case of his works on mental language, for instance, which bring him closer to certain theories elaborated by Hilary Ptnam, especially his theory of represtation.

KEY WORDS - Medieval philosophy. Ockham. Putnam. Mental language. Verbum mentis. Thought acts. Theory of representation. Direct knowledge.

\section{Dal verbum mentis al linguaggio del pensiero}

L'idea che esista una forma particolare di linguaggio, accanto al linguaggio orale e scritto, una forma di linguaggio che sia propria degli esseri immateriali e quindi anche della parte spirituale dell'uomo, cioè della sua mente, ha attraversato tutto il pensiero cristiano da s. Agostino e Boezio fino alle elaborate dottrine sul linguaggio degli angeli, esseri dotati di pura forma e quindi di solo spirito, proprie dei secoli XIII e XIV.

Agostino aveva postulato l'esistenza di un verbum interior nell'ambito del problema della Trinità:

"chiunque [...] può comprendere che cosa sia il verbo, non soltanto prima che risuoni al di fuori, ma anche prima che il pensiero si occupi delle immagini dei suoni; questo verbo infatti non appartiene ad alcuna lingua, a nessuna di quelle che chiamano 'lingue delle genti', fra le quali c'è anche la nostra lingua latina [.....".1

* Università di Padova, Italia.

Cfr. De Thinitate 15, 11, 19: "Quisquis igitur potest intelligere verbum, non solum antequam sonet, verum etiam antequam sonorum eius imagines cogitatione volvantur; hoc enim est quod ad nullam pertinet linguam, earum scilicet quae linguae appellantur gentium, quarum nostra latina est [...]".

\begin{tabular}{|l|l|l|l|l|l|}
\hline VERITAS & Porto Alegre & v. 45 & n. 3 & Setembro 2000 & p. $349-360$ \\
\hline
\end{tabular}


"Perciò il verbo che risuona al di fuori è segno del verbo che risplende all'interno e che, più di ogni altro, merita tale nome di verbo."

Per Agostino la necessità di un verbo interiore (verbum mentis) nasceva dalla constatazione che

“[...] tutte queste conoscenze che l'anima umana acquisisce da sé e per mezzo dei sensi del corpo e per testimonianza degli altri, le tiene riposte nel tesoro della sua memoria; sono esse che generano un verbo vero, quando diciamo ciò che sappiamo, verbo che precede ogni parola che risuona e ogni pensiero della parola che risuona. Allora infatti il verbo è perfettamente simile alla cosa conosciuta da cui nasce e di cui è immagine, perché dalla visione della scienza procede la visione del pensiero, che è un verbo non appartenente a nessuna lingua, verbo vero da una cosa vera, che non possiede niente di proprio, ma riceve tutto da quella scienza da cui ha origine". ${ }^{3}$

La studiata contrapposizione tra cogitatio soni, un pensiero ancora legato alla sua espressione orale, e verbum mentis, parola della mente, nelle intenzioni di Agostino è sufficiente per fondare l'autonomia di questo tipo di linguaggio da ogni elemento sensibile; ma Agostino non riesce a liberarsi completamente neanche in questo caso dalla sua concezione del linguaggio come imago e come vestigium delle cose denominate e perciò finisce per concepire il verbum mentis ancora in senso neo-platonico come la capacità di richiamare alla mente, di ricordare appunto.

Boezio a sua volta teorizza una oratio mentalis o cogitabilis in accordo con le dottrine degli aristotelici piuttosto che in riferimento ai succitati testi di Agostino:

"è stato affermato dai Peripatetici che vi sono tre tipi di discorso: uno che è scritto con le lettere, un altro che è pronunciato mediante la voce e un terzo che si trova congiunto nell'animo. Ora se vi sono tre tipi di discorso, non vi è dubbio che anche le parti del discorso sono di tre tipi. Dal momento che il verbo e il nome sono le parti principali del discorso, vi sarà un tipo di verbi e di nomi che saranno scritti e un altro tipo che saranno trattati dalla mente silenziosa."

Gli autori medievali, in ogni caso, troveranno chiaramente teonizzate anche nei suoi testi le basi per elaborare un complesso linguaggio mentale, quale strumento indispensabile per arrivare alla conoscenza delle cose esteriori. Ma sia la concezione agostiniana, costruita sul concetto di imago e di vestigium, come quella aristotelicoboeziana, basata sulle passiones animae, comportano inevitabilmente, come osserva Ockham,

2

3

Cfr. De Trinitate XV, XII, 22: "Haec igitur omnia, et quae per se ipsum, et quae per sensus sui corporis, et quae testimoniis aliorum percepta scit animus humanus, thesauro memoriae condita tenet, ex quibus gignitur verbum verum, quando scimus loquimur, sed verbum ante omne sonum, ante omnem cogitationem soni. Tunc enim est verbum simillimum rei notae, de qua gignitur, et imago eius, quoniam de visione scientiae visio cogitationis exoritur, quod est verbum linguae ullius, verbum verum de re vera, nihil de suo habens, sed totum de illa scilicet de qua nascitur." Si veda anche XV, X, 19.

4 Cfr. Boezio, In Perihermeneias II, p. 30, 3-10: "Dictum est tres esse apud Peripateticos orationes, unam quae litteris scribitur, aliam quae profertur in voce, tertiam quae conjungeretur in animo. Quod si tres orationes sunt, partes quoque orationis triplices esse nulla dubitatio est. Quare quoniam verbum et nomen principaliter orationis partes sunt, erunt alia verba et nomina quae scribantur, alia quae dicantur, alia quae tacita mente tractentur". 
"che la cosa rappresentata abbia bisogno di essere conosciuta in precedenza, altrimenti il rappresentante non porterebbe mai alla conoscenza dell'oggetto rappresentato come a qualcosa di simile"

e il logico medievale la illustra con grande chiarezza facendo riferimento alla relazione che esiste tra Ercole e la sua statua, sulla traccia di una analogia già ampiamente in uso presso gli autori medievali:

"è evidente in base all'esperienza che se qualcuno, che non sappia nulla di Ercole, vede una sua statua non avrà alcun motivo di pensare ad Ercole piuttosto che a Socrate. Se invece costui ha prima la possibilità di vedere Ercole e conserva una qualche notizia di Ercole e solo dopo vede una statua a lui simile per alcune caratteristiche esteriori, per il semplice fatto di vedere la statua, anche se prima non l'aveva mai vista, si ricorderà di Ercole [...]. Lo stesso accade per il vestigium, per cui se qualcuno vede il vestigium di un bue si ricorderà del bue che già conosce abitualmente. Ma se invece non avesse avuto prima una qualche notizia relativa al bue, non avrà alcun motivo di ricordarsi di un bue piuttosto che di un asino.. ${ }^{5}$

Questo tipo di conoscenza può solo condurci a ricordare a che cosa somigli un certo oggetto, ma non può mai condurci ad una nuova conoscenza delle caratteristiche proprie dell'oggetto.

Ma Ockham supera tutte queste forme di conoscenza attingendo nel linguaggio mentale la funzione specifica del significare:

"il termine 'segno' viene assunto in un modo più specifico per designare ciò che fa venire a conoscenza di qualcosa e che per sua natura può stare al posto di tale cosa nella proposizione o esservi aggiunto [...] o che per sua natura può essere composto di tali cose, quale è l'enunciato [...]".

La lingua nella quale si esprime immediatamente questa funzione significativa naturale è rappresentata proprio dal linguaggio mentale. II linguaggio mentale, in effetti, è costituito da puri concetti, anzi da concetti ridotti nella loro funzione essenziale di segni delle cose, cioè alla loro pura capacità referenziale. Tali concetti significano direttamente e naturalmente le cose o i vari stati di cose e consentono che le differenti lingue storicamente diversificate possano venire tradotte reciprocamente, cioè garantiscono la comunicazione tra gli uomini che parlano lingue diverse. In tal modo tutte le lingue storicamente date costituiscono l'espressione

Cfr. Reportatio II,, 12-13, p. 274, 7-8: “[...] repraesentatum debet esse prius cognitum, aliter repraesentans nunquam duceret in cognitionem repraesentati tanquam in simile [... $]^{n}$; d. 3, q. 9, p. 575, 8 : "per experientiam enim patet quod si aliquis nullam penitus habeat cognitionem de Hercule, si videat statuam Herculis non plus cogitabit de Hercule quam de Sorte. Si autem primo videat herculem, et retineat notitam Herculis, et postea videat statuam sibi similem quantum ad aliqua accidentia exteriora, virtute illius visionis statuae, etiam posito quod nunquam prius eam vidisset, recordabitur de Hercule, quamvis non semper, propter imperfectionem similitudinis vel propter aliquem alium defectum. Et ita est de vestigio, quod si aliquis videat vestigium bovis recordabitur de bove habitualiter cognito, sed si nunquam prius habuisset aliquam notitiam de bove non plus recordaretur de bove quam de asino".

Summa logicae I, 1, p. 9, 59-64: "Aliter accipitur signum pro illo quod aliquid facit in cognitionem venire et natum est pro illo supponere vel tali addi in propositione ... vel quod natum est componi ex talibus, cuiusmodi est oratio". 
ridondante dell'unico linguaggio mentale che appartiene a tutti gli uomini allo stesso modo. Nel linguaggio mentale noi troviamo tutte quelle strutture linguistiche dei linguaggi storici che sono rilevanti ai fini della determinazione della verità e della falsità delle proposizioni; pertanto non vi saranno sinonimi, termini equivoci ed espressioni logicamente superflue proprie di ogni lingua storica e riconducibili all'esigenza di ornare il proprio modo di esprimersi. Si tratta quindi di un linguaggio universale, naturale, ideale e perfetto perché solo in questo linguaggio la necessitas significationis, cioè la funzione segnica propria di quei segni che sono le parole, può esprimersi allo stato puro, senza le interferenze e le ambiguità del linguaggio storico.

\section{Caratteristiche del linguaggio del pensiero}

II linguaggio del pensiero è strutturato allo scopo di stabilire la verità o la falsità degli enunciati senza le ambiguità proprie del linguaggio parlato e scritto. Tali ambiguità - secondo Ockham - dipendono dalla natura convenzionale di ogni linguaggio parlato e scritto:

"si deve sapere che solo un enunciato o un altro segno stabilito per convenzione è equivoco o univoco. Perciò, una intenzione dell'anima o concetto, non è né equivoca, né univoca propriamente parlando"?

Il linguaggio mentale, al contrario, non è basato su una convenzione, ma unicamente sulla necessitas significationis del segno e quindi in esso non compaiono né termini equivoci, né termini univoci, né sinonimi:

"la moltiplicazione dei sinonimi non è stata inventata a causa della necessità di significare, ma per abbellire il discorso o per qualche altra causa secondaria, dal momento che tutto ciò che può essere significato per mezzo di tutti quei sinonimi può venire espresso per mezzo di uno solo di essi e quindi a tale pluralità di sinonimi non corrisponde affatto una moltitudine di concetti".

Cfr. Summa logicae I, 13, p. 44, 10-12: "Est autem primo sciendum quod sola vox vel aliud signum ad placitum institutum est aequivocum vel univocum, et ideo intentio animae vel conceptus non est aequivocus nec univocus proprie loquendo".

8 Summa logicae I, 2, p. 11, 18-22 : "[...] sicut nominum synonymorum multiplicatio non est propter necessitatem significationis inventa, sed propter ornatum sermonis vel aliam causam consimilem accidentalem quia quidquid per omnia synonyma significatur posset per unum illorum exprimi sufficienter, et ideo multitudo conceptuum tali pluralitati synonymorum non correspondet". Si veda anche: Quodlibeta V, q. 8, p. 510, 66-69: "[...] multitudinem talium accidentium quae competunt nominibus synonymis non oportet tribuere naturalibus signis cuiusmodi sunt conceptus mentales, sicut nec nominibus synonymis correspondet pluralitas conceptuum"; Summa Logicae I, 3, p. 12, 45-51: "[...] accidentia nominibus propter necessitatem significationis non conveniunt [...] et aliquando accidit quod duo nomina sunt synonyma et tamen sunt generum diversorum et aliquando diversarum figurarum, propter quod talem multiplicitatem non oportet naturalibus signis tribuere. Unde quaecumque pluralitas et varietas talium accidentium quae potest competere nominibus synonymis, potest convenienter a mentalibus amoveri". 
Naturalmente si potrebbe obiettare che quanto viene sostenuto da Ockham riguarda unicamente i termini del linguaggio mentale e non le proposizioni che pure si possono formare in tale linguaggio. Non è difficile, in effetti, proporre delle proposizioni composte da termini totalmente esenti da ambiguità, ma tali da risultare al contrario profondamente ambigue tanto da poter essere analizzate con differenti condizioni di verità.

In effetti, al riguardo la posizione di Ockham non sembra essere molto chiara poiché egli è convinto che sia sufficiente non avere termini ambigui per non avere proposizioni ambigue, poiché per lui ogni proposizione è il risultato di una aggiunta di varie parti del discorso. Egli riconosce che "[...] esiste la proposizione mentale, poiché ovunque si trovi un complesso vero o falso vi è una proposizione, ma nella mente esiste un tale complesso, quindi esiste la proposizione mentale",, ma il suo criterio per la costituzione dei complexa mentalia è lo stesso dei complexa vocalia o scripta, cioè il cosiddetto principio di addizione. In base a tale principio una proposizione significa esattamente la somma totale di ciò che significano i suoi termini categorematici.

\section{Dalla critica alla teoria della rappresentazione agli atti di pensiero}

La critica della teoria del concetto come rappresentazione, termine che dovrebbe venire inteso nel suo senso etimologico di ri-presentazione o nuova presentazione dell'oggetto, si fonda sostanzialmente sul fatto che, se ogni oggetto deve essere "rappresentato", cioè riportato alla memoria, per essere conosciuto, noi non arriveremo mai alla conoscenza. In effetti, anche la "rappresentazione dell'oggetto" per essere conosciuta dovrebbe, a sua volta, essere sottoposta ad una ri-presentazione e cosi all'infinito.

Per evitare questa situazione paradossale Ockham aderì per un qualche tempo alla teoria del fictum. Ockham, pur mantenendo la sua avversione verso ogni concetto universale, ammette per un certo tempo l'esistenza di entità fittizie singolari nell'intelletto per spiegare il processo conoscitivo. Questi oggetti fittizi avrebbero "una esistenza oggettiva nello stesso senso in cui le cose rappresentate hanno una esistenza soggettiva", nel senso che un ens fictum che esiste oggettivamente nella mente di chi conosce nel momento in cui conosce, sarebbe simile alle cose esterne che rappresenta nel caso in cui fossero dotate di una esistenza puramente soggettiva. Con "esistenza oggettiva" di tali entia ficta si intende che essi costituiscono il vero oggetto che viene conosciuto, qualunque sia il tipo di esistenza di cui sono dotati.

Ockham spiega questa particolare natura dei ficta con un esempio:

Cfr. Quodilibeta III, 12, p. 247, 13-15: “[...] probo quia ubicumque est complexio vera vel falsa, ibi est compositio; sed in mente est huiusmodi complexio; igitur etc. [...] cuilibet complexioni in voce correspondet aliqua complexioni in mente [...]". 
"[...] si può dire che l'intelletto nell'apprendere un oggetto singolare si costruisce fittiziamente un altro oggetto singolare della stessa natura e tale oggetto singolare cosi costruito non esiste realmente in nessun altro luogo, come non esiste realmente, prima di averlo effettivamente costruito, il castello che l'ingegnere progetta e tuttavia nella sua realtà progettuale è esattamente come nella realtà esterna". ${ }^{10}$

Viene naturale osservare come in questo caso Ockham abbia finito per tradire doppiamente i suoi assunti metodologici sulla ammissione di entità non necessarie: i ficta sono per definizione realtà fittizie e non reali e tuttavia si pretende che in questa loro esistenza fittizia siano dotati di una natura oggettiva, cioè costituiscano i veri oggetti della conoscenza o i contenuti proposizionali.

Ma sembra si possa spiegare l'atteggiamento del grande logico medievale in altro modo. Innanzitutto, Ockham non sembra voler applicare il suo principio di parsimonia verso entità che non hanno una natura reale, come sono i ficta. Tutto il suo sforzo era andato nella direzione di vietare che gli enti di ragione venissero presentati come reali. Evidentemente egli non considera una violazione del suo rasoio l'ammissione di entità fittizie che non comportano un impegno ontologico e la cui natura viene lasciata nell'ambiguità degli enti dotati di un ens diminutum, cioè di una esistenza di tipo inferiore. Inoltre, l'accettazione della teoria dei ficta sembra rappresentare per lui una tipica strategia razionale di natura temporanea, per poter arrivare alla soluzione definitiva. In effetti, sembra che Ockham abbia aderito alla teoria del fictum come a una mossa strategica per poter confutare le forme di realismo radicale nella spiegazione della conoscenza che ponevano nelle cose stesse o una natura communis o comunque degli aspetti oggettivi rappresentati nell'unità del concetto. Una volta ottenuto lo scopo avrebbe abbandonato anche la teoria dei ficta che gli aveva permesso di criticare le teorie contranie alla sua.

Stabilendo definitivamente che il concetto è una intentio è possibile trasferire tali aspetti comuni dagli oggetti conosciuti alla nostra mente conoscente. E' il soggetto conoscente che costituisce gli aspetti comuni che danno unità al concetto, ma questi non si trovano nella realtà esterna e non hanno nemmeno un fondamento reale in essa, ma sono solo costruzioni della mente.

Peraltro la teoria del fictum, pur preziosa nella critica ad ogni forma di realismo, finiva per dare una autonomia tale al concetto da renderlo oggetto di definizione reale e ciò significava che il concetto da solo non era più in grado di svolgere la funzione per la quale era stato elaborato, cioè di spiegare il riferimento alle entità singolari, le uniche ad essere ritenute veramente reali. In effetti, se il concetto, inteso come una rappresentazione fittizia delle cose, fosse un essere oggettivo, con un proprio contenuto, esso non potrebbe più essere lo strumento adatto per significare tutti i singolari che cadono sotto di esso:

${ }^{10}$ Cfr. Ordinatio I, 2, 8, p. 279, 20-23: "Ex hoc patet quod talia ficta sunt talia in esse obiectivo qualia sunt alia in esse subiectivo, et sic intellectus haberet virtutem productivam, faceret ea esse similia in esse subiectivo"; Expositio in lib. Penhermenias I, prooem. 7, p. 359, 13-360, 17: "Et per hunc modum potest dici quod intellectus apprehendens singulare fingit consimile singulare et illud singulare sic fictum non est alicubi exsistens realiter; non plus quam castrum quod artifex fingit exsistit realiter antequam producat ipsum, et tamen est tale in esse ficto quale est aliud extra". 
"coloro che pongono degli esseri fittizi diranno che quando è conosciuto l'universale, non è conosciuta alcuna cosa esteriore"." ${ }^{11}$

In tal modo la teoria della rappresentazione come fictum risultava incapace di spiegare il riferimento dei concetti agli esseri singolari, gli unici esseri ad esistere veramente e perciò falliva il suo compito.

In base a questa considerazione Ockham nelle sue ultime opere abbandona completamente la teoria del fictum perché gli risulta essere del tutto inutile per portare a compimento la conoscenza dei singolari e pertanto, invece, di risolvere il problema della conoscenza finisce per renderlo più complicato. Ancora una volta ci si trova di fronte ad un tertium che impedisce il rapporto diretto tra conoscente e cosa conosciuta, la cui natura è estremamente ambigua. Egli non esita ad abbandonare questo "parvus mundus" fatto di entità così strane come i ficta:

"non vi sono esseri oggettivi di questo genere, cioè che non sono, né possono essere enti reali; non ha senso ammettere un piccolo mondo differente per questo tipo di esseri oggettivi; ciò che non è alcuna cosa non esiste affatto". ${ }^{12}$

La vicenda dei ficta, da un certo punto di vista, può rappresentare il massimo avvicinamento di Ockham alla teoria delle distinzioni formali di Scoto nella ricerca di entità che non sono né reali, nè di ragione. Ma Ockham si rende conto che la conoscenza può essere spiegata solo mediante il riferimento diretto alle cose singolari e che pertanto la natura del concetto non può che essere costituita da un essere reale e non fittizio e che il riferimento stesso va posto nei suoi elementi essenziali, cioè in un rapporto reale tra soggetto e oggetto all'interno di una pura funzione segnica.

\section{Riferimento diretto e atti di pensiero}

In realtà, solo trasformando la gnoseologia, teoria del concetto, in semiologia, teoria del segno, Ockham può risolvere in maniera soddisfacente il problema conoscitivo. I concetto, pertanto, viene spogliato dalle ambigue e complicate costruzioni mentali che lo avevano gravato da quando Aristotele aveva parlato dei concetti come di pathemata en the psyché e la tradizione medievale, ad iniziare da Boezio, si era a lungo interrogata sulla natura di tali passiones animae, intendendole ora come immagini e memorie conservate dalla fantasia, ora come concetti dotati di una propria realtà. ${ }^{13}$

${ }^{11}$ Cfr. Ordinatio I, d. 27, q.3, p. 255, 14-16: "[...] qui ponerent entia ficta [...] dicerent quod quando intelligitur universale, nulla res extra intelligitur. Quando tamen intelligitur singulare sive notitia intuitiva sive abstractiva, intelligitur singulare".

12

Cfr. Quodlibeta III, 4, pp. 218-219: "Ad tertium dico quod non sunt talia esse obiectiva, quae non sunt nec possunt esse entia realia; nec est unus parvus mundus alius entium obiectivorum; sed illud quod nulla res est, omnino nihil est".

13 E' noto che Boezio tradusse il termine aristotelico presente nel passo del De interpretatione I, 16a38 "le cose nel linguaggio sono simboli $(\sigma u \mu \beta 0 \lambda \alpha)$ delle passioni dell'anima", con "notae". Aristote$\mathrm{le}$, in effetti, aveva usato anche on $\mu \varepsilon i \alpha$, "segni" nello stesso contesto. $\dot{\mathrm{E}}$ evidente che il primo termine rinvia ad una natura diversa di tale passio animae, rispetto alle cose esteriori mentre il secondo si limita a considerarle come note distintive. Sembra che Boezio usando il termine "nota", "segno distintivo", abbia volutamente mescolato i significati dei due termini greci. Boezio, per la verità, intende in ogni caso le "notae in anima" senz'altro come intellectus, cioè come entità astratte, ma tutta la disputa sugli universali sta a testimoniare la possibilità di intenderle anche come puri segni. 
Ockham interpreta l'espressione aristotelica en the psyché non in senso metaforico, ma in senso reale, cioè nel senso che nella mente è iscritto realmente qualcosa: ma per lui l'unico modo di scrivere qualcosa realmente nella mente è quello del linguaggio dei segni, che sono reali, come vedremo, e che tuttavia danno origine ad una operazione immateriale come quella del significare.

Ockham, in effetti, deve spiegare come un atto della mente sia in grado di stabilire un riferimento diretto alle cose singolari che esistono fuori della mente. Egli procede per gradi dimostrando prima di tutto che un unico atto interno è in grado di fare riferimento ad un numero infinito di oggetti singolari:

"che l'intenzione concettuale faccia riferimento a un numero infinito di singolari non è meno accettabile che si possano amare o desiderare con lo stesso atto d'amore o di desiderio un numero infinito di cose; infatti, qualcuno può amare tutte le parti di un continuo che sono infinite oppure può desiderare che tutte le parti di un continuo perseverino nell'essere e tuttavia questo appetito non riguarda che una certa parte del continuo e non una più dell'altra. Pertanto è necessario che tutte siano desiderate anche se sono infinite [...]. Allo stesso modo si può dire che uno stesso atto conoscitivo può riguardare una serie infinita di cose senza avere una relazione esclusiva con una sola di tali cose infinite, né senza distinguere un atto da un altro a causa di una certa somiglianza speciale dell'atto conoscitivo con quelli individui e non con altri." ${ }^{14}$

Quindi il logico medievale stabilisce che l'atto intellettivo, inteso come segno delle cose singolari, è qualcosa di reale anche se è una pura intentio della mente, si tratta cioè di una qualità reale, allo stesso modo in cui sono reali i segni scritti con l'inchiostro su un foglio di carta:

"bisogna sapere che secondo coloro che pongono l'intenzione, il concetto o la passione nell'anima è una qualità dello spirito ed essi non la chiamano 'essere di ragione' perché non sarebbe una cosa veramente esistente nella natura delle cose, ma perché è solo nella ragione e la mente ne fa uso al posto di un'altra cosa o in forza di un'altra cosa [...] dunque, la divisione fatta da Averroè e da Aristotele tra l'essere reale e l'essere di ragione o l'essere nell'anima e fuori dell'anima non è una divisione stabilita tra realtà direttamente opposte $[\ldots] .{ }^{.15}$

${ }^{14}$ Cfr. Expositio in lib. Perihermenias, p. 355, 97-: "[...] nec magis videtur hoc [ SC. quod actus apprehendendi sive sciendi unam propositionem est aliquis unus actus simplex] esse inopinabile quam quod eadem dilectione vel desiderio possunt infinita diligi vel desiderari. Sed hoc secundum non videtur inopinabile, nam potest aliquis diligere omnes partes alicuius continui quae sunt infinitae, vel potest appetere quod omnes partes continui durent in esse, et tamen non appetitur esse tali appetitu nisi aliqua pars continui, et non magis una quam alia. Oportet quod omnes appetantur, quae tamen sunt infinitae [...]. Sic igitur posset dici quod eadem cognitio potest esse infinitorum, non tamen enit cognitio propria alicui illorum, nec ista cognitione potest unum distingui ab alio et hoc propter aliquam similitudinem specialem istius cognitionis ad individua illa et non alia".

Cfr. Summa logicae I, 40, p. 113, 60-69: "[...] sciendum est quod secundum opinionem quae ponit quod intentio, conceptus sive passio animae est qualitas mentis, non ideo dicitur aliquid 'ens rationis' quia non sit vera res exsistens in rerum natura, sed ideo dicitur ens rationis quia non est nisi in ratione, quo mens utitur pro alio vel propter aliud [...] ideo quod Commentator et Philosophus dividunt ens primo in ens reale et in ens rationis, sive in ens in anima et in ens extra animam [...] non est divisio per simpliciter opposita [..." 
L'atto intellettivo è, pertanto, qualcosa di reale esattamente come è reale il bianco che dipingiamo su un muro:

"ritengo che colui che vuol sostenere questa posizione dovrebbe affermare più opportunamente che tutte le proposizioni, i sillogismi e ogni intenzione dell'anima e in generale tutto ciò che è detto ente di ragione sono veri enti reali, esistenti positivamente e sono qualità della mente che determinano realmente la forma della mente, così come il bianco determina la forma della parete e il calore determina la forma del fuoco [... $]^{16}$

Per Ockham pertanto parlare nel linguaggio mentale costituisce una operazione analoga a quella di porre del colore su una parete, cioè si tratta in ambedue i casi di una qualità reale capace di trasformare in atto qualcosa che lo era solo in potenza. Non si tratta, però, di dipingere nella mente una immagine o una rappresentazione dell'oggetto. L'atto stesso del conoscere diventa simile alla cosa conosciuta mediante il processo referenziale proprio del segno. Esattamente come ogni segno è in qualche modo ciò che significa, analogamente l'atto intellettivo è in modo puramente referenziale la cosa conosciuta:

"prima dell'atto intellettivo non è richiesta alcuna previa assimilazione per mezzo di una species, ma è sufficiente l'assimilazione che avviene per mezzo dell'atto intellettivo che è esso stesso una somiglianza della cosa conosciuta. Secondo Agostino, quando si conosce qualcosa direttamente, allora l'intellezione sarà molto simile alla cosa $\mathrm{e}$ non richiede altra somiglianza oltre alla intellezione". ${ }^{17}$

Stabilita la natura del concetto come atto di conoscere ne consegue anche il riferimento diretto alle cose singolari e l'esistenza di qualcosa di reale nella mente del conoscente. Tutto questo, inoltre, è un processo naturale che si svolge necessariamente in base alla necessitas significationis propria di ogni segno naturale. In questo modo il concetto inteso come segno è dotato di una sua propria unità che non va più cercata a parte obiecti in una natura communis o nelle rationes formales positivae di Duns Scoto, ma che è posta a parte subiecti nell'atto stesso di intendere e pertanto è solo una unità di significazione, cioè la capacità di cogliere la serialità che vi è nelle cose, o, per così dire, la capacità di cogliere l'accadere proprio dei fenomeni secondo una somiglianza posta dalla ragione.

${ }_{16}$ Cfr. Expositio in lib. Perihermenias I, prooemium, p. 358, 200-205: "Et qui vult tenere istam opinionem reputo quod magis dicet convenienter si dicat quod omnes propositiones, syllogismi, quaecumque intentiones animae, et universaliter omnia quae vocantur entia rationis, sunt vere entia realia positiva et verae qualitates mentis realiter informantes mentem, sicut albedo informat realiter parietem et calor ignem".

Cfr. Reportatio II, q. 13, pp. 295-296: “[... d]ico quod non requiritur ante actum intelligendi aliqua assimilatio praevia quae sit per speciem. Sed sufficit assimilatio quae fit per actum intelligendi qui est similitudo rei cognitae. Quia secundum Augustinum, $V$ De Trinitate, quando aliquid intelligitur ut est in se, tunc intellectio erit simillima rei, et non requiritur praeter intellectionem alia similitudo". 


\section{L'Ercole di Ockham e la formica di Putnam}

E' straordinario trovare una conferma della correttezza del percorso seguito da Ockham nella critica alla rappresentazione e alla successiva elaborazione della teoria degli atti intellettivi in un filosofo contemporaneo tra i più noti, Hilary Putnam. Se Ockham aveva utilizzato l'esempio della statua di Ercole per introdurre le sue argomentazioni contro la dottrina della species, Putnam arriva alle stesse conclusioni seguendo il tracciato di una formica sulla sabbia:

"Una formica cammina su una spiaggia di sabbia e, camminando, traccia una linea sulla sabbia. Per caso, la linea da essa tracciata fa una curva a un certo punto e, tornando indietro, incrocia se stessa parecchie volte, sino a divenire una caricatura riconoscibile di Winston Churchill. La formica ha tracciato un'immagine di Winston Churchill, un'immagine che lo rappresenta?"

Come nel caso della statua di Ercole scoperta da chi non sa nulla di Ercole, neanche "la rappresentazione di Churchill" da parte della formica, che a maggior ragione non sa nulla di questo grande statista, non è in grado in se stessa di far sorgere in noi alcun concetto relativo all'oggetto rappresentato, per cui bisogna concludere che "la linea [ma lo stesso vale per una statua] non è in 'se stessa' una rappresentazione di una cosa piuttosto che di qualsiasi altra [...] la rassomiglianza non è sufficiente [...] la rassomiglianza non è neppure necessaria [...]". ${ }^{19}$

Nell'analisi proposta da Putnam intesa a "negare connessioni necessarie fra le rappresentazioni e i loro riferimenti", Putnam ripercorre in parte la via seguita da Ockham e conclude con il logico medievale non solo che "se esistono rappresentazioni mentali che si riferiscono necessariamente (a cose esterne), esse devono essere della medesima natura dei concetti, e non di quella delle immagini", ma anche che "i concetti sono segni usati in un certo modo [...] anche se il segno indipendentemente dal suo impiego non è il concetto [...] i segni da soli non si riferiscono intrinsecamente a nulla" ${ }^{20}$

In tal modo Putnam perviene alla mental act theory per spiegare i concetti esattamente come Ockham, dopo un lungo percorso, era giunto alla teoria dell'actus intelligendi.

Non vi è dubbio che, nonostante la distanza temporale, i testi dei due pensatori siano in grado di illuminarsi a vicenda ben oltre le rassomiglianze finora suggerite. Bastino le seguenti osservazioni. Putnam ci dà una ragione fondamentale per capire come le "rappresentazioni mentali", in quanto tali, non siano né sufficienti, né necessarie per la elaborazione dei concetti, per il semplice fatto che i concetti "non sono presentazioni mentali", ma piuttosto "la capacità del pensante di impiegare questi fenomeni [interni], di usare il fenomeno giusto nelle circostanze giuste". 
A sua volta Ockham, utilizzando una concezione semiotica molto scaltrita, sembra andare oltre questa indicazione di "impiego" che Putnam ripropone sotto l'influsso di Wittgenstein, e preferisce parlare di actus, e precisamente di "actus intellectivi", ben consapevole che nel processo semiotico qualcosa accade, peraltro senza dare origine a nuove entità, cioè viene posto un riferimento puramente intenzionale. Solo intendendo l'intenzionalità in questo modo, cioè come processo segnico, è possibile eliminare veramente quel terzo elemento, comunque venga inteso, che la tradizione filosofica da Aristotele in avanti aveva postulato per spiegare la conoscenza del mondo esterno.

Ora, tutti i maggiori pensatori contemporanei ricordano che l'attuale filosofia della mente continua a combattere contro "l'idea disastrosa" che ha ossessionato la filosofia occidentale fin dal secolo XVII, cioè l'idea che la percezione ha bisogno di una interfaccia tra la mente e gli oggetti "esterni" che noi percepiamo. ${ }^{21}$

Questa "disastrosa idea", che si impose in maniera così radicale grazie al dualismo cartesiano in tutta la filosofia occidentale, è ancora lungi dall'essere abbandonata sia in filosofia che nelle neuroscienze, dove molti fenomeni della coscienza vengono ancora spiegati con il ricorso alla teoria del cosiddetto "omuncolo" (homuncolus theory), un piccolo uomo collocato dentro il cervello che è capace di vedere nella mente come se questa fosse una specie di schermo televisivo interno.

Non vi è dubbio, che se la vicenda storica di Ockham avesse avuto un destino differente e se la sua filosofia avesse ottenuto l'attenzione che meritava, la conoscenza della mente umana avrebbe potuto disporre di una teoria dei processi conoscitivi assai più avanzata e la prospettiva materialista difficilmente sarebbe stata introdotta nello studio della coscienza.

\footnotetext{
${ }^{21}$ Cfr. H. PUTNAM, The Face of Cognition, in Sense, Nonsenses, and the the Sense: An Inquiry into the Powers of the Human Mind, "The Journal of Philosophy", 91(1994), p. 488: "[...] our difficulty in seeing how our minds can be in genuine contact with the 'extemal' world is, in large part, the product of a disastrous idea that has haunted Western philosophy since the seventeenth century, the idea that perception involves an interface between the mind and the 'external' objects we perceive".
} 